\title{
QUANTUM MAGNETOTRANSPORT IN InGaAs/InP AT HIGH MAGNETIC FIELDS AND LOW TEMPERATURES
}

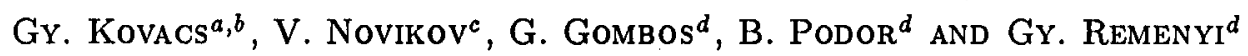

a CNRS Centre de Recherches sur les Tres Basses Temperatures, Grenoble France

${ }^{b}$ Department of Low Temperature Physics, Eotvos Lorand University Budapest, Hungary

${ }^{c}$ A.F. Ioffe Physico-Technical Institute of the Russian Academy of Sciences St. Petersburg, Russia

${ }^{d}$ Research Institute for Technical Physics of the Hungarian Academy of Sciences Budapest, Hungary

Quantum Hall effect and Shubnikov-de Haas effect measurements were made in two-dimensional electron gas in liquid phase epitaxially grown $\mathrm{Ga}_{0.47} \mathrm{In}_{0.53} \mathrm{As} / \mathrm{InP}$ heterostructures in high magnetic fields in the temperature range from $4.2 \mathrm{~K}$ down to $60 \mathrm{mK}$. Two-dimensional electron gas concentrations and mobilities were in the range of $(1-3) \times 10^{11} \mathrm{~cm}^{-2}$ and $(1-3) \times 10^{4} \mathrm{~cm}^{2} /(\mathrm{V} \mathrm{s})$, respectively. Corresponding to this the $i=1$ quantum Hall effect plateau occurred at about $6 \mathrm{~T}$ magnetic field. Although fractional occupation numbers of about 0.3 were reached, no signs of fractional quantization were detected. Both current and frequency breakdown of the quantum Hall effect were investigated. Narrowing of the plateaus with increasing current differs from that measured in $\mathrm{GaAs} / \mathrm{AlGaAs}$ structures because of the different mechanisms of dissipation. The fact that the magnetic length becomes smaller than the characteristic scale of the disorder seems to be essential in understanding the low frequency breakdown via the presence of quasi-classical electron states.

PACS numbers: 73.20.Dx

\section{Introduction}

It is well known [1] that the main scattering process in InGaAs/InP heterostructures is the alloy disorder scattering. In the case of MOCVD grown samples $[2,3]$ the role of scattering on the interface roughness is debated, however, liquid-phase epitaxy (LPE) results in heteroboundaries with marked and reproducible spatial fluctuations [4-7]. Various techniques (microscopic ellipsometry, mobility analysis, weak localization experiments and Shubnikov-de Haas (SdH) measurements) have revealed a small scale fluctuation of the profile of the heterointerface with a characteristic height of $0.7-1.0 \mathrm{~nm}$ and period of 5 to $10 \mathrm{~nm}$. 
A large scale roughness having a period of about $100 \mu \mathrm{m}$ and amplitude of $50 \mathrm{~nm}$ was also detected. This is a quite new feature compared to the widely studied MBE grown GaAs/AlGaAs structures. In the latter samples the disorder is not smooth, the magnetic length is comparable or greater than the characteristic length of the disorder potential. Theoretically it was suggested [8] that when magnetic length becomes smaller than the disorder length, the frequency dependence of the quantum Hall effect (QHE) plateaus occurs at the $\mathrm{kHz}$ range, contrasted to the usual hundreds of $\mathrm{MHz}$ domain. At the same time, the mechanism of the (corner) dissipation [9] is via the scattering on the smooth disorder. Measuring the length of the QHE plateaus in function of the current, a relation, different from the GaAs/AlGaAs case, is expected.

\section{Experimental}

The LPE InGaAs/InP samples consisted of three layers on the (100) semi-insulating InP:Fe substrate. The layer sequence was the following: a $1 \mu \mathrm{m} p$-type InP buffer with $p=10^{15} \mathrm{~cm}^{-3}$, a $0.2-0.5 \mu \mathrm{m} n$-type InP layer with $n=(2-10)$ $\times 10^{16} \mathrm{~cm}^{-3}$ and a $0.5-1.5 \mu \mathrm{m} p$-type $\mathrm{Ga}_{0.47} \mathrm{In}_{0.53} \mathrm{As}$ layer with $p \approx 10^{15} \mathrm{~cm}^{-3}$. In order to make the $p$-type layers electrically purer, they were doped with $\operatorname{Sm}[10,11]$.

Two-dimensional electron gas (2DEG) concentration varied between $1.1 \times 10^{11} \mathrm{~cm}^{-2}$ and $3.4 \times 10^{11} \mathrm{~cm}^{-2}$. A comparison between the concentration values obtained from the classical Hall data and those from the $\mathrm{SdH}$ oscillations demonstrated that no parallel conduction was present. Fourier transform of the oscillations reveals one component, indicating that only one subband was populated. Magnetotransport measurements were carried out in a dilution refrigerator down to $60 \mathrm{mK}$. Three kinds of techniques were used: DC transport measurements, standard lock-in experiments and a special lock in a technique using the so-called ORPX Siemens-meter. Lock-in frequency varied up to the hundred $\mathrm{kHz}$ range while frequency of the ORPX was fixed at $23 \mathrm{Izz}$.

\subsection{Electron heating and current breakdown of $Q H E$}

From the analysis of the $\mathrm{SdH}$ amplitudes it is clear that electron heating can be neglected only for currents much smaller than $1 \mu \mathrm{A}$, even at the helium temperature. The resolution of the spin splitted oscillations is dramatically improved at low ( $\mathrm{nA}$ ) currents and low temperatures.

We have studied the current breakdown in terms of the width of the QHE plateaus in function of the current. It is known that the dependence of the length of the QHE plateaus on the current strongly depends on the dominant scattering mechanism leading to dissipation. Usually scaling is assumed and a power-like relation is expected. According to the results shown in Fig. 1 in our case this assumption is forced and experimental data fit to an exponential. Noteworthy to mention that the exponent does not depend on the plateau index, suggesting that its value is determined by the dissipation process.

In our preliminary calculations we have adopted the model which assumes that the electron heating is due to the injection of hot electrons from the contact region. The electric field at those regions is greater than the breakdown field, thus 
QHE conditions are violated. The injected electrons dissipate via scattering on the smooth disorder (i.e. alloy and interface roughness scattering).

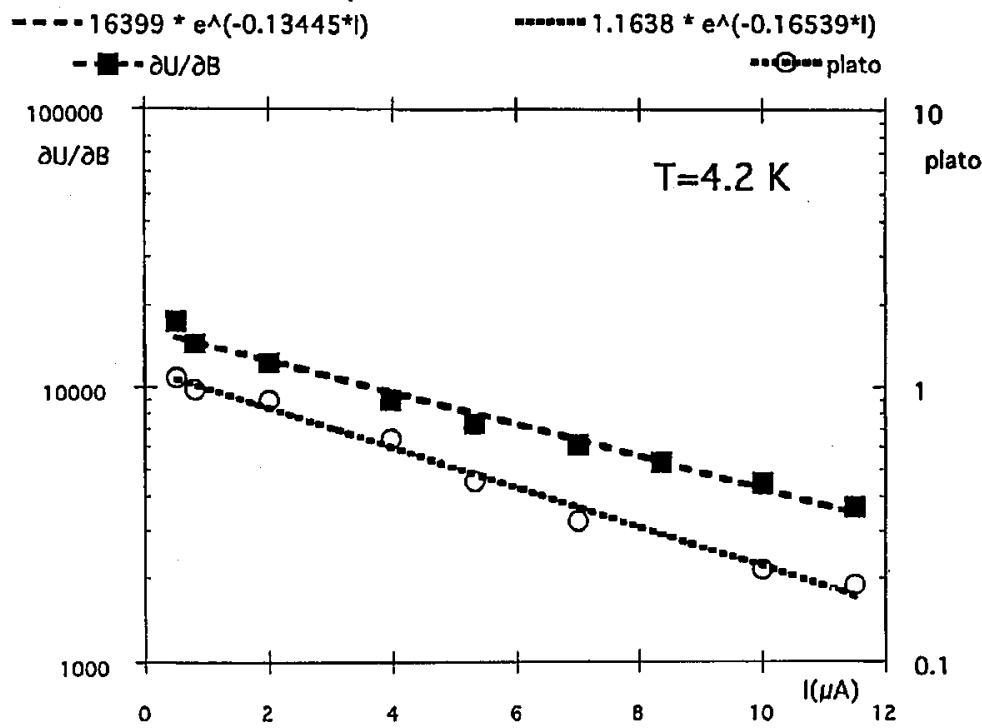

Fig. 1. Width of plateau vs. current. Determination of the width was done using two definitions: first - the magnetic field interval in which the Hall resistance is equal to the theoretical value $\pm 0.3 \%$; second - with the help of the derivative of the Hall resistance with respect to the magnetic field.

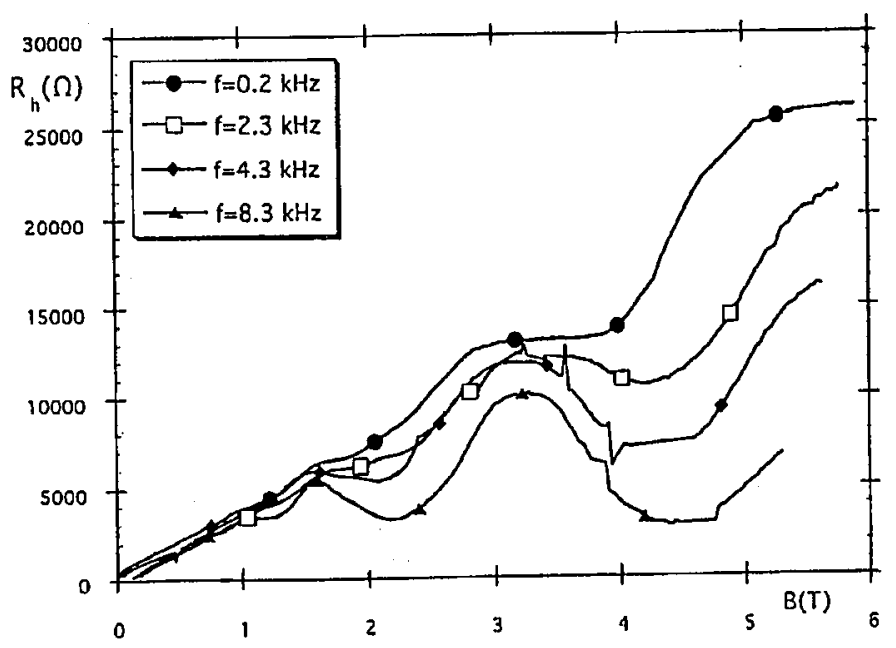

Fig. 2. Frequency breakdown of QHE. Note that the breakdown occurs at the $\mathrm{kHz}$ frequency range, indicating the role of the quasi-classical states. 
It is worth to mention that while increasing the current the phase of the $\mathrm{SdH}$ oscillation is changed above a certain magnetic field, i.e. at a given $B$ corresponding to a SdH minimum for low currents there is a $\mathrm{SdH}$ maximum for higher currents. This results is an unstable current-voltage characteristics above that magnetic field.

\subsection{Frequency breakdown of $Q H E$}

Figure 2 shows that regular QHE plateaus are distorted in the $\mathrm{kHz}$ frequency range. This observation seems to contradict the fact that QHE is destroyed only in the hundred $\mathrm{MHz}$ range. However, recently it was suggested [5] that the breakdown frequency should depend on the ratio of the magnetic length to the characteristic scale of the disorder, and when this value is smaller than unity, the breakdown frequency is reduced. Our observations are in good qualitative agreement with that prediction, based on a percolation theory in the presence and in the absence of quasi-classical orbits.

\section{Summary}

Various phenomena related in part to the smooth disorder potential were observed in our preliminary observations. In spite of the theoretical possibility, no signs of fractional quantization were observed.

This work was supported in part by the Hungarian National Research Foundation (grant No. OTKA/12037). In Grenoble Gy. K. was supported by a TEMPRA grant while G. G. received a financial support from the French Ministry of Education. Gy. K. acknowledges the kind hospitality of the SNCI-MPI. Valuable discussions with I.G. Savel'ev are deeply appreciated.

\section{References}

[1] W. Walukiewicz, H.E. Ruda, J. Lagowski, H.C. Gatos, Phys. Rev. B 30, 4571 (1984).

[2] M. Razeghi, J.P. Duchemin, J.C. Portal, L. Dmowski, G. Remenyi, R.J. Nicholas, A. Briggs, Appl. Phys. Lett. 48, 825 (1986).

[3] S. Ben Amor, L. Dmowski, J.C. Portal, K.P. Martin, R.J. Higgins, M. Razeghi, Appl. Phys. Lett. 57, 2925 (1990).

[4] B. Pdor, S.V. Novikov, I.G. Savel'ev, G. Gombos, Acta Physica Hung. 74, 143 (1994).

[5] S.D. Bystrov, A.M. Kreshchuk, S.V. Novikov, T.A. Polyanskaya, I.G. Savel'ev, Fiz. Tekhn. Poluprov. 27, 645 (1993).

[6] L.V. Golubev, A.M. Kreshchuk, S.V. Novikov, T.A. Polyanskaya, I.G. Savel'ev, I.I. Saidashev, Fiz. Tekhn. Poluprov. 22, 1948 (1988).

[7] S.D. Bystrov, A.M. Kreshchuk, Le Tuan, S.V. Novikov, T.A. Polyanskaya, I.G. Sa vel'ev, A.Ya Shik, Semiconductors 28, 55 (1994).

[8] R. Joynt, J. Phys. C 18, L331 (1985).

[9] J. Riess, P. Magyar, Acta Phys. Pol. A 84, 523 (1993).

[10] V.V. Vorob'eva, M.V. Egorova, A.M. Kreshchuk, S.V. Novikov, I.G. Savel'ev, I.I. Saidashev, Pis'ma Zh. Tekhn. Fiz. 15, 73 (1989).

[11] N.A. Bert, V.V. Vorob'eva, M.V. Vorontsova, A.M. Kreshchuk, S.V. Novikov, K.Yu. Pogrebitskii, I.G. Savel'ev, D.Zh. Saifidinov, I.P. Soshnikov, A.Y. Shik, Fiz. Tekhn. Poluprov. 24, 653 (1990). 